II. Aus der chirurgischen Klinik des Herrn Geh.-Rath Prof. Dr. Bardeleben in Berlin.

\title{
Ueber die chirurgische Behandlung der narbigen Pylorusstenose.
}

Von Stabsarzt Dr. A. Koehler.

Innere Medicin und Chirurgie haben nirgends so viele Berührungspunkte uud sind anf keinem Gebiete so sehr zu gemeinsamer Arbeit berufen, wie auf dem der Erkrankung der Unterleibsorgane. Diese, in grossen Krankenhäusern, in denen innere und chirurgische Abtheilungen neben einander stehen, schon lange bekannte und gewürdigte Thatsache verdient es um so mehr, in ärztlichen Kreisen immer wieder betont zu werden, als das Zusammenwirken der Vertreter beider Theile der heilenden Kunst bereits eine ganze Reihe schöner Erfolge aufzuweisen hat.

Die innere Medicin hat dabei hauptsächlich durch Vervollkommnung der Untersuchungsmethoden und der gerade auf diesem Gebiete so schwer genau zu stellenden, und doch für den Erfolg maassgebenden Diagnose, die Chirurgie durch die Fortschritte in Antiseptik und Aseptik und durch die Verbesserungen in der operativen 'l'echnik mitgewirkt.

Es ist nur ein kleiner Abschnitt des grossen und in letzten Jahrzehnt vollständig umgearbeiteten Capitels der Bauchchirurgie, für welches ich heute Ihre Aufmerksamkeit in Anspruch nehme, es ist aber ein Abschnitt, in welchem eine Reihe sehr guter Resultate zu verzeichnen ist.

Zu ihnen gehört auch der Frfolg in einem, von Geh. Rath Bardeleben am 4. Februar d. J. operirten Falle von Pylorusstenose.

16 Jahre altes, hisher gesundes, aber immer schwächliches Mädchen, hat angeblich in ihrer Kindleeit einmal "Franzbranutwein mit Labestock" (?) getrunken. Seit October 1889 Nagenschmerzen, Erbrechen, Abmagerung. Aufnahme anf der I. medicin. Klinik am 15. Januar 1890. Colossale Ausdelmung des Magens (untere Grenze in der Mitte zwischen Symphyse nud Xabel), Erbrecheu alles Genossenen, U rinmenge $200 \mathrm{~cm}$ pro Tag. Bei Magenansspullungen und ernährenden Klystiren keine Besserung; darum. Verlegung zur chirurgischen Klinik am 4. Februar. An demselben 'Tag Operation: Magenausspülung, antiseptische Reinigung. Ruhige Chloroformbetänbung. Sclnitt in der Lin. alba vom Proc. xiph. $7 \mathrm{~cm}$ nach abwärts. Der Pylorus war leicht hervorzuziehen und fïhlte sich an, wie ein mässig harter Strang. Aensserlich war weder an ilım, noch an den angrenzenden Theilen von Duodenum und Nagen etwas Abnormes zı sehen. Duodenum und Magen wurden von einem Assistenten mit den Fingern abgeklemmt, dẹr Pylorus der Länge nach gespalten, nachdem man sich überzeugt hatte, dass die Fingerspitze mit der eingestülpten Magenwand nicht in ihn einzufïhren war. Durch Auseinanderziehen der Wundränder in ihrer Nitte wurde die Längswunde quergestellt und so vereinigt, eine Nahtreihe (feine Seide) durch die ganze Dicke der Wand, eine zweite (auch Seide) durch die Serosa nach Lembert. Schluss der Bauchwunde, indem Peritoneum für sich, dann die beiden Recti, die oberflächliche Fascie und die Ilant, jedes fïr sich, mit Catgut vereinigt wurden. In die Hautwunde wurden noch 4 Seidenknopfnähte eingelegt. Dauer bis zum Anlegen der Bauchnaht ${ }^{3 / 4}$ Stunden. Der Jodoformgazewatteverband blieb 8 Tage liegen; als er dann zum ersten Mal gewechselt wurde, war die ganze Wunde per primam verheilt. In den ersten 10 Tagen exnälrende Klystire; dabei aber vom dritten Tage an Lismilch, dann Bouillon mit Ei, Schabefleisch, Haferschleim. Die Kranke fühlte sich wohl, hatte keine Magenbeschwerden bis zım 1\%. Tage, wo sie eine nicht genau zu bestimmende Quantität eingeschmuggelter Wurst gegessen hatte. Jetzt trat wieder heftiges Erbrechen ein, welches sich nach 4 Tagen, dann 
nach 3 Tagen, dann ungefähr jeden zweiten Tag wiederholte. Die Urinmenge war dabei auf $1000-1200 \mathrm{ccm}$ täglich gestiegen; die Kranke fühlte sich wohl; jedenfalls ein Beweis, dass die Naht gut gehalten hatte. Weil das Erbrechen nicht nachliess, wurde die Patientin am 12. März zur I. medicinischen Klinik zurückverlegt. Auch hier änderte sich der Zustand nur wenig, so dass die Nothwendigkeit einer zweiten Operation in Erwägung gezogen und die Patientin am 27. März der chirurgischen Klinik wieder überwiesen wurde. Unter specieller Leitung und Fürsorge des Stationsarztes (Stabsarzt Muhlack) wurde die bisherige Behandlung versuchsweise fortgesetzt und erzielte eine stetige, wenn auch langsame Besserung. Das Körpergewicht del Patientin, welches am 29. März $53^{3} / 4$ Pfd. betrug, ist auf $671 / 2$ Pfd. gestiegen, die Urinmenge normal geworden; in der letzten Zeit ist sogar Polyurie (täglich ca. $3000 \mathrm{ccm}$ normaler Beschaffenheit) eingetreten. Die Kräfte haben sich ganz bedeutend gehoben, so dass wir der Kranken wohl eine definitive Heilung ohne Operation in Aussicht stellen können. Das Aushebern 4 Stunden nach de Mahlzeit wird noch fortgesetzt. Die Patientin führt sich die Sonde mit Leichtigkeit selbst ein.')

In einem 2. Falle, den Oberstabsarzt R. Köhle r am 15. April operirte, lagen die Verhältnisse viel schwieriger und complicirter.

Die 31 Jahre alte Patientin hatte am 1. Mărz Schwefelsäure getrunken und wurde am 2. April von der I. medicinischen zur chirurgischen Klinik verlegt, weil die Zeichen der Verengerung des Pylorus immer mehr zunahmen. Die Urinmenge schwankte zwischen 200 und $700 \mathrm{ccm}$ pro Tag, war aber meistens noch über $500 \mathrm{ccm}$. Die Kranke wog $82 \mathrm{Pfd}$. - Es ist mindestens unsicher, ob es besser gewesen wäre, sie sofort zu operiren; die Erwägung, dass eine grössere Quantität Schwefelsäure getrunken war, dass die Pat. am 8. Tage nach der Aufnahme einen ca. $13 \mathrm{~cm}$ im Durchmesser haltenden Schleimhautfetzen erbrochen hatte, der Ihnen seiner Zeit vom Stabsarzt Brettner demonstrirt wurde, dass also auf ausgedehnte Zerstörungen gerechnet werden musste, welche in 4 Wochen kaum geheilt sein konnten, hielt uns davon ab. Am 15. April, also 6 Wochen nach dem Selbstmordversuch, wurde die Operation vorgenommen. Die Kranke war ganz sicher in ihrem Kräftezustand nicht mehr heruntergekommen, als die oben besprochene Pat., und als der im Juli 1888 von Geh.-Rath Bardeleben operirte Patient (s. Tab. No. 7). _.- Vorbereitungen, wie in dem 1. Falle. Schnitt in der Linea alba. Der Pylorus fühlte sich hart, knollio an und war $4 \mathrm{~cm}$ breit mit einer Dünndarmschlinge durch feste Stränge verwachsen, aber doch leicht vorzuziehen. Von einer in der Nähe des Pylorus angelegten Magenwunde aus war die Strictur auch für Sonden nicht durchgängig; von einer Wunde im Duodenum, welche zum Theil in den Pylorus selbst hineinging, gelang die Erweiterung des Restes der Strictur bis zum Durchführen von 2 Fingern. Naht der Magenwunde durch 2 Reihen Seidennähte; Nalit der Wunde im Duodenum und Pylorus zum grösseren Thei nach $\mathrm{Heineke,} \mathrm{zum} \mathrm{kleineren} \mathrm{in} \mathrm{der} \mathrm{Längsrichtung,} \mathrm{Naht} \mathrm{der} \mathrm{Bauchwunde}$ mit Seide. Von der Eröffnung der Bauchhöhle bis zum Verschluss derselben waren doch $2^{1 / 2}$ Stunden (10 Uhr 25 Minuten bis 1 Uhr 10 Minuten) vergangen; die Narkose war ruhig, es waren im ganzen nur $45 \mathrm{~g}$ Chloroform verbraucht. Am Nachmittag schlief die Kranke viel, Puls 120; kein Erbrechen. Nach einer ziemlich unruhigen Nacht trat Morgens 7 Uhr plötzlich Collaps und Tod ein. Bei der Section wurde in den geoffneten Oesophagus Luft eingeblasen, welche den Magen, das Duodenum und einen Thei des Dünndarmes stark aufblähte, ohne dass aus den Nahtstellen Luft entwichen wäre. Der Pylorus war bequem für einen Finger durchgängig. Keine Spur von Peritonitis.

Die bisher ausgeführten Fälle von Pyloroplastik sind in folgender Tabelle zusammengestellt:

Tabelle über die bis jetzt ausgeführten Operationen der narbigen Pylorusstenose durch Pyloroplastik.

\begin{tabular}{|c|c|c|c|c|c|}
\hline No. & Quelle & $\begin{array}{l}\text { Datum } \\
\text { und Chirurg }\end{array}$ & $\begin{array}{l}\text { Alter } \\
\text { d. Pat. }\end{array}$ & Indication & Ausgang \\
\hline
\end{tabular}

1. Frohnmüller, $\mathbf{1} 886$ Heineke, März Frau, Narbe eines Ge- Heilung (noch \begin{tabular}{l|l|l|l} 
Dissertał. Fürth 1886. & $34 \mathrm{~J}$. & schwürs. & jetzt nach $4 \mathrm{~J}$
\end{tabular} und briefl. Mit-

theilung an den festgestellt!)

Verf.

2. Archivf.klin.Chir. Mikulicz, Feb. Frau, Narbe u. Geschw., Tod (an ErBd. 37 Heft 1. ruar $1887.20 \mathrm{~J}$. unstillibaresBlut- schöpfung $\mathrm{n}$

3. Contributo alla Novaro, Jan. brechen. 3 Tagen). Chirurgia dello 1888 . $34 \mathrm{~J}$. (Narbe?) Plastik rerBlutung n. \begin{tabular}{ll|l|l|l|} 
Stomaco Siena. & und Loreta. & 5 Tagen).
\end{tabular}

Briefliche Mitthei- Heineke, 3. Mann Strictur am Pylor. Heilung. (Tod Brieflich an den Verf.
lung Juni 1888. $\quad \begin{gathered}\text { Strictur am Pylor. Heilung. (Tod } \\ \text { im Duodenum. }\end{gathered}$ ten an Tuberculose).

5. Ortmann, deutsche Mikulicz, 25. Mäd- Narbe, 6 J. nach Heilung (nach med. Wochen- Juni 1888. chen, dem Trinken 1 ca. $1 \frac{1}{2}$. Jahre schrift 1859 , No.

9 und briefl. Mit theil. an den Verf

6. S. No. 3! Novaro, 30. Frau, Fibröse Entartung Heilung (noch \begin{tabular}{|l|lll}
\hline Juni 1888. & $44 \mathrm{~J}$ & und Polypen. & jetzt nach 2
\end{tabular}

1) Die Kranke ist, nachdem 4 Wochen lang keine Magenausspülungen mehr nöthig gewesen waren, Mitte August geheilt entlassen. Körpergewicht 75 Pfund.

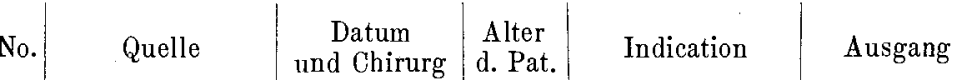

7. A. Köhler, Berli. Bardeleben 25. Maun, Narbe, $7^{1 / 2}$ Wochen Heilung (Tod nerklin.W ochen- Juli 1888 . $35 \mathrm{~J}$. nach dem Trinken nach 5 Mon. schrift 1888, No. $\quad$ eines Schluckes an Tubercu46.

8. Archiv für klin. v, d. Hoeven, Mann, Narbe, 8 Wochen Heilung (noch Chir., Bd.38, Heft 1. Aug. 1888. 29 J. nach demTrinken jetzt nach fast 2 und briefl. Mittheil. an den Verf.

9. S. No. 3 und 6 11. Novaro, 7. Feb- Mann, Fibröse Entartung Heilung (März briefl. Mitheil. ruar $1889 . \quad 35 \mathrm{~J} . \quad$ (Narbe). 1890 Recidiv.

S. Novaro 1. c. p. Carle (Turin). 14.

11. 18. Congr. d. deut- Lauenstein,28. schen Gesellsch. März 1889. für Chirurgie und briefl. Mittheil. an den Verf.

12. S. No. 3, 6 u. 9. Novaro, 17.De- Manu, Fibröse Entartung Heilung (noch

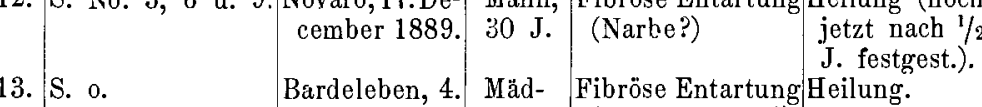

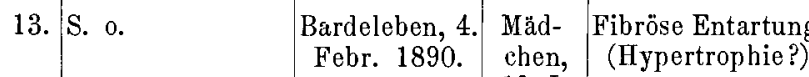
14. S. No. 3, 6 u. 12. Novaro, 20. Mann, Narbe und Ge-Heilung (noch Febr. 1890. 20 J. schwür. jetzt nach 4 Mon. festgestellt).

15. Brief. Mittheil. Novaro, 13. 16. S. 0 . R. Köhler, 15. Mäd- Narbe, 6 Woclıen Tod (nach 18 April 1890. chen, nachdemTrinken Stunden im 31 J. von Schwef.l- Collaps). säure (Plas

Die narbigen Verengerungendes Pylorus, welche in der Mehrzahl der Fälle nach dem Trinken ätzender Flüssigkeiten, seltener im Verlaufe der Vernarbung eines aus anderer Ursache in der Nähe des Pylorus entstandenen Geschwüres sich bilden, sind von der sogenaniten Hypertrophie des Pylorus schwer zu unterscheiden, bei welcher sich keine eigentliche Narbe, sondern ein fast solider Ring mit sehr kleiner centraler Oeffnung in der Pylorusgegend vorfindet. Oft wird es sich doch um Vernarbungsprocesse handeln - die Patienten geben nicht $z u$, dass sie vor längerer Zeit einen Selbstmordversuch gemacht haben - oft genug muss man Reizungszustände in der Pylorusgegend annehmen, welche, ähnlich den krampfhaften, durch kleine Schrunden verursachten Zusammenziehungen des Sphincter ani, allmählich zu einer Hypertrophie, vielleicht auch nur zu einem schwer zu überwindenden Krampf und schliesslich zu fibröser Degeneration der Ringmuskulatur führen. Ebenso schwer kann es werden, die skirrhöse Form des Carcinoms am Pylorus von einer einfachen narbigen Velengerung zu unterscheiden. Die Symptome können dieselben sein, das Fehlen der freien Salzsäure im Mageninhalt hat auch nicht immer nachgewiesen werden kőnnen. Hier köunte nur eine nach Freilegung und Spaltung des Pylorus sofort vorgenommene mikroskopische Untersuchung Gewissheit verschaffen und für das weitere Handeln maassgebend werden. $O b$ einer der seltenen Fälle von angeborener Pylorusstenose, über welche $H$. Landerer im Jahre 1879 in seiner Dissertation (Freiburg) schrieb, operirt ist, konnte ich nicht ausfindig machen; vielleicht handelt es sich bei der sogenanten Hypertrophie des Pylorus zuweilen um Verschlimmerung angeborener Zustände. Schliesslich kann eine Verengerung, ein Verschluss des Pylorus, wie jeder anderen Stelle des Darmcanals, auch durch Entzündungen verursacht werden, welche nicht in ihm (Ulc. rotundum, tuberculos., syphilitic.), sondern an ihm, im Peritoneurn der Nachbarschaft verliefen und zu Strangbildungen und Verziehungen, Abknickungen führten. Die letztgenannten Fälle, sowie die therapeutisch ganz anders zu beurtheilenden Verengerungen durch gutartige und bösartige Geschwülste werden im folgenden nicht berücksichtigt werden.

Von den Operationen, welche im allgemeinen für die Verengerung des Pylorus empfohlen und geübt sind, kommt bei der einfachen narbigen Stenose, bei welcher die angrenzenden Theile von Magen und Zwölffingerdarm gesund sind, und bei welcher der verengte Pylorus, frei von entzündlichen Verwachsungen, sich leicht in die Bauchwunde hineinziehen lässt, nur die Pyloroplastik in Frage. Ganz gewiss sind eine Reihe guter Frfolge dabei mit der Resection des verengten Pylorus, noch mehr mit der Anlegung einer Magendünndarmfistel, der Jejunogastrostomie, erzielt. Beide Operationen sind aber so viel mühsamer und dauern so viel 
länger, als die Heineke'sche Pyloroplastik, dass man diese vorziehen wird, um so mehr, als sie bis jetzt mindestens keine schlechteren Resultate aufzuweisen hat, als die genannten Operationen. Ein leichterer Eingriff scheint auf den ersten Blick in der Loreta'schen Operation zu liegen, in dem stumpfen Erweitern der verengten Stelle mit dem durch eine Magenwunde eingeführten Finger, wie es Loreta im Jahre 1882, ohne von dem Vorschlage Richter's (Breslau) Kenntniss zu haben, zuerst ausführte. Diese Divulsio digitalis stösst bei narbigen Stenosen oft auf so grosse Schwierigkeiten, erfordert eine solche Kraft, dass es fast unmöglich erscheint, sie ohne Schleimhautrisse, deren Tiefe sich nicht vorhersagen lässt, auszuführen. Eine Wunde in der Nähe des Pylorus muss dabei auch angelegt werden, denn die intacte Magenwand in den narbigen Pförtner so fest hineinzudrängen, dass die Narbe nachgiebt, würde an zwei Stellen starke Quetschungen setzen. Es ist deshalb gewiss besser, von vornherein die Wunde im Pylorus anzulegen, diesen der Länge nach zu spalten, den Zustand der Schleimhaut und der verengten Stelle genau zu untersuchen, und nöthigenfalls, wenn die Möglichkeit eines Skirrhus vorliegt, ein kleines Stäckchen zur milkroskopischen Untersuchung auszuschneiden. Die Längswunde, welche rechts und links bis in die gesunde Wand des Duodenum und des Magens reicht, wird dann durch Auseinanderziehen der Wundränder in ihrer Mitte quer gestellt und so durch mehrere Nahtreihen vereinigt. Die frühere Narbe bildet dann die Hinterwand des neuen Canals, dessen vordere Wand von den, von rechts und links herbeigezogenen gesunden 'Theilen des Zwölffingerdarmes und Magens gebildet wird. Man wird gut thun, für die tiefe, die Schleimhant mitfassende Nahtreihe resorbirbare Fäden zu nehmen; die zweite, Serosanaht, hat sicher zu vollständiger Verklebung geführt, wenn die inneren Nähte resorbirt sind. Die Losstossung von Seidenfäden aus der Schleimhaut in den Canal hinein wird wahrscheinlich viel langsamer erfolgen und leichter zu kleinen Substanzverlusten führen. - Die Erweiterung mit dem Finger würde wahrscheinlich, wie Novaro betont, auf die Fälle von Stenose durch Hypertrophie zu beschränken sein und hier ebeuso leicht gelingen, wie die Dehnung anderer Sphincteren. Liesse sich die Differenzialdiagnose zwischen narbiger und durch Hypertrophie bedingter Verengerung mit Sicherheit stellen, dann wäre dieser Ansicht beizupflichten; das ist aber nicht immer der Fall. Da nun ganz entschieden davor gewarnt werden muss, bei narbigen Stenosen mehr, als ganz vorsichtige Versuche der Dilatation mit Instrumenten und Fingern zu machen, so würde man oft in die Lage kommen, nach der Loreta'schen noch die Heineke'sche Operation machen zu müssen - gewiss eine nicht ungefährliche Verlängerung der Operationsdauer für den fast immer äusserst schwachen Patienten. Die Erweiterung mit dem Finger bei narbigen Verengerungen hat ausserdem in mehreren Fällen (Loreta selbst und Falleroni, s. Navaro, l. c.) wiederholt werden müssen, weil sich ein Recidiv einstellte; Codivilla (Gazz. di osp., No. 75 und 78, 1888) berichtet über dasselbe Ereigniss bei Hypertrophie des Pylorus. Trotzdem konnte Barton im Mai 1889 schon 25 Fälle von Operation nach Loreta zusammenstellen; ja Loreta selber hat die Dilatation $30 \mathrm{mal}$ ausgeführt und kannte im ganzen 43 Fälle. Dass die Operation mit der Zeit an Gefahr verloren hat, beweist Barton dadurch, dass er für die ersten 12 Fälle eine Mortalität von $50 \%$, für die letzten 12 eine Mortalität von $25 \%$ ausrechnet. Im letzten Jahre ist sie noch $4 \mathrm{mal}$ mit günstigem Erfolge ansgeführt (Treves, Kinnicut und Bull, Huntington und Gardner). Die Pyloroplastik ist, soweit ich habe sammeln können, bisher 16 mal ausgeführt, bei 7 Männern und 7 Frauen resp. Mädchen, (2 unbestimmt, Tab. No. 10 und 15). Bei den 7 \%wischen 20 und 35 Jahre alten Männèrn 2 mal wegen narbiger Stenose nach Anätzungen, $4 \mathrm{mal}$ wegen fibröser Verầnderung der Muscularis am Pylorus, ohne dass anamnestisch die Anätzung festgestellt werden konnte, und $1 \mathrm{mal}$ bei Ulcus rotundum. Bei den weiblichen Patienten im Alter zwischen 15 und 42 Jahren handelte es sich 2 mal um Aetznarben, 2 mal um Ulcus und 3 mal um die anderen genannten Veränderungen. Von den 16 Fällen sind 11 (1 Fall von Novaro, s. u., ist des Recidivs verdächtig) als geheilt zu betrachten; ich rechne dazu auch den 2. Fall von $\mathrm{Heineke}$ und die beiden von Bardeleben operirten Fälle. Der 2. Patient Heineke's (in der Liste No. 4) starb 2 Monate nach der leicht überstandenen Operation an schon vorher nachgewiesener Tuberculose. "Seit der Operation waren Störungen von Seiten des Magens nicht mehr dagewesen, und Patient vertrug die seinem Zustande angemessene Kost, ohne wieder zur Magensonde greifen zu müssen". Der erste Patient Bardeleben's (Liste No. 7) starb im Krankenhause Friedrichshain 5 Monate nach der Operation, ebenfalls an Tuberculose. Ich rechne auch diesen Fall zu den durch Plastik geheilten Pylorusstenosen, weil der Patient in den 5 Monaten nie wieder Magenbeschwerden gehabt hat. 6 Wochen nach seiner Eutlassung, also 3 Monate nach der Operation, habe ich ihn in seiner Kellerwohnung am Ostbahnhof besucht; er hatte einen kleinen Kohlenhandel und trug die nicht leichten Körbe treppauf und -ab, ass nach seiner Aussage ganz wie früher und klagte nur über seinen hartnäckigen Katarrh. Eine Magenerweiterung war damals nicht nachzuweisen. Dass die Plastik in diesem Falle wirklich zur Heilung geführt hat, beweist aber auch das Präparat, welches uns Herr Geh. Kath Hahn freundlichst überlassen hat; erstens sind, wie Klemperer Ihnen das vor 1 Jahre demonstrirt hat, die Magenwände wieder vollständig normal, die Ektasie ist geheilt; und zweitens ist der Pylorus, obgleich das Präparat jetzt $1^{1 / 2}$ Jahre in Spiritus liegt, für den Zeigefinger bequem durchgängig. (Es war also ein Irrthum von Ewald, wenn er behanptete, die Verengerung sei in diesem Falle wieder eingetreten; s. Deutsche medicinische Wochenschrift Nr. 23, 1889.) Der 2. Bardeleben'sche Fall, die Patientin, welche heute vorgestellt wurde, muss wegen ihrer hartnäckigen Magenektasie, welche sie seit der Operation $2 \mathrm{mal}$ durch schwere Diätsünden wieder verschlimmert hat, noch behandelt werden (s. o. Anmerk.), sie zeigt aber seit Monaten eine ununterbrochene deutliche Besserung: Zunahme des Körpergewichts in 2 Monaten um 14 Pfund, normale Urinmenge und eine beträchtliche Verkleinerung der Magenektasie. - Von den anderen Geheilten lebt die "erste Pyloroplastik" noch heute, also nach 4 Jahren, in bestem Wohlsein. Prof. Heineke schreibt am 5. d. M.: "Hente Abend stellte sich mir die Person wieder vor. Sie sieht gesund und kräftig aus und hat eine nicht unerhebliche Körperfülle. Sie fühlt sich vollkommen gesund, kann alle Speisen vertragen und schont den Magen in keiner Weise. Als fleissige Wäscherin ist sie alle Tage thätig." Dr. van der Hoeven hat auf meine Bitte seinen Patienten am 7. Juni zu sich bestellt (fast 2 Jahre nach der Operation) und schreibt: „Er war ganz gesund und sehf kräftig und hatte seit der Operation gar keine Magenbeschwerden mehr'gehabt." Geh. Rath Mikulicz hat die letzte Nachricht über seine Patientin im Herbste 1889 , also über 1 Jahr nach der Operation gehabt; damals war "das Befinden in jeder Richtung befriedi-

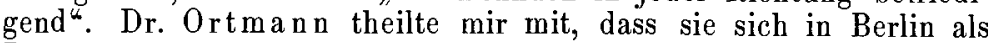
Dienstmädchen vermiethet habe; hier war ihre Wohnung nicht zu ermitteln. Novaro schreibt Mitte Juni: "Von den 4 Geheilten befinden sich drei in dem Augenblicke, in welchem ich schreibe, vortrefflich. Der vierte (auf den sich die Krankengeschichte 10 bezieht) klagt neuerdings über Magenbeschwerden; ich fürchte, dass es sich um ein Carcinom handelt."

Diesen 11 Heilungen stehen 4 Todesfälle nach der Operation gegenüber - auf den ersten Blick eine schlechte Empfehlung. Betrachtet man aber diese Fälle etwas genauer, dann stellt sich heraus, dass es sich bei zweien (Liste No. 3 und 16) gar nicht um einfache Plastik gehandelt hat, sondern dass erst Versuche gemacht waren, die Stenose mit Sonde und Finger zu erweitern. Novaro (l. c. p. 64) fand als Todesursache eine innere Blutung aus den, bei der Divulsion gesetzten Schleimhautrissen; in dem anderen Falle war davon nichts zu sehen; die Nähte hielten vollkommen dicht. Die durch die vorausgeschickte Loreta'sche Operation bedingte Verlängerung des ganzen Eingriffs wird bei der schwachen Patientin den schlimmen Ausgang, wenigstens zum Theil, verschuldet haben. - In dem ersten Falle von Mikulicz handelte es sich um eine, durch häufiges Blutbrechen aus einem $2 \mathrm{~cm}$ in den Pankreas hineinragenden Magengeschwür auf's äusserste heruntergekommene Frau, welche sich nach der Operation nur vorübergehend erholte und am 3. Tage im Collaps starb. Bei der Section zeigte sich der neue Pylorus genügend weit; die Nähte hielten vollkormmen dicht. - Noch viel complicirter und schwieriger lagen die Verhältnisse in dem Falle Lauenstein's.

Die 42 Jahre alte Patientin hatte seit 2 Jahren an Magenbeschwerden, seit 14 Tagen an Erbrechen gelitten. Es fand sich ein wenig verschieblicher, nach links von der Mittellinie zwischen Nabel und Proc. ensitormis gelagerter Tumor von Gänseeigrösse, mit dem längsten Durchmesser quergestellt. Nahm die Patientin nur flüssige Speisen, so erbrach sie nicht. Der Magen war sehr klein und fasste nur 1/4 I Flüssigkeit. Ich stellte die Diagnose auf ein Ulcus der kleinen Curvatur nale dem Pylorus, welcher jedoch noch durchgängig sein musste. Bei der am 28. März 1889 vorgenommenen Operation fand ich nach Eröffnung des Leibes in der Mittellinie den Pylorns des kleinen Magens in ein daumendickes, starres Rohr verwandelt, von dem aus sich ein über fingerdicker harter; tumorartiger. Strang der kleinen Curvatur entlang nach links und oben erstreckte. Das Duodenum Iang ausgezogen und von normalem Aussehen. Da bei der eigenthümlichen Kleinheit und der nach aufwärts und nach links fixirten Lage des Magens eine Pylorusresection unmöglich gewesen wäre und eine Gastroenterostomie weder vollkornmen indicirt, noch überhaupt ausführbar war, so unternahm ich eine Plastik des nach hinten nicht fixirten Pylorus nach Heineke-Mikulicz. Es wurde, vom Duodenum beginnend, ein $8 \mathrm{~cm}$ langer Längsschnitt durch die vordere Wand der Pylorusgegend gemacht. Die Magenwandung zeigte sich stark verdickt. und starr, sowie ăusserst brüchig, so dass eine isolirte Vereinigung der Schleimhautränder in dem verzogenen Spalt misslang. Die hyperämische Schleimhaut war so brüchig und mürbe, dass die Fāden, beim Versuche, sie za knüpfen, sofort durchschnitten. Da auch ein Einstülpen der brüchigen, starren Magenwand unmörlich war, so musste Wundrand gegen Wundrand genāht werden, und die Nahtstiche durchdrangen die ganze Dicke der Wand. 
Dabei musste, um ein Durclsschneiden der Fäden zn verhindern, noch ganz vorsichtig geknipft werden. Nachdem die Nahtlinie nit einer feinen Lage Jodoform bestäıbt war, wurde der Leib geschlossen. - Danter der Operation 48 Minnten. Am Abend des Operationstages hatte die Patientin 100 Pulso nnd 24 Athmungen, war an Händen ınd Füssen warm, fühlte sich wohl nnt liatte nicht erbrochen. Sie erbielt 3 Tage lang keine Nahrung durch den Mund, sondern wurde mit Kklystiren ernährt. Am 31. März bekam sie zalerst etwas flinssige Nalırung. Die Temperatur stieg schon denselben Abend auf 38,4, am 1. April auf 39,0, dazu gesellte sich heftiger Leibsclumerz, und es trat am Morgen des 2. April der Exitus ein. - Die Section ergab, dass aus einigen Stichcanälen Mageninlaalt ausgetreten und dadurch eine septische Peritonitis in der Ungebung der Nahtlinie verursacht war.

Wenn man die beiden Fälle, in denen die Loreta'sche und Heineke'sche Operation gemacht war, bei der Betrachtung der Mortalität fortlässt, dann bleiben 2 Todesfälle auf 14 Fälle von Pyloroplastik; aber auch diese beiden sind weniger der Operation als solcher zur Last zu legen, als der Schwere der Inanition in dem ersten, und der starken, ausgedehnten Erweichung (der Schnitt in die verengte Stelle musste $8 \mathrm{~cm}$ lang angelegt werden) der Magenwand in dem zweiten Falle. - Wir sehen deshalb von einer Berechnung der Sterblichkeit nach Procenten ab; eine solche hat für die geringe Zahl von 16 Fällen überhaupt wenig Werth. - Die Pyloroplastik leistet bei der narbigen Pylorusstenose jedenfalls mehr, als die Loreta'sche Operation; sie stellt nahezu normale Verhältnisse wieder her und ist leichter und schneller anszuführen, als die ilır sonst gleichwerthigen Operationen, die Resection des Pylorus und die Jejunogastrostomie. Von dem Anlegen einer Düundarmfistel (also Ausschluss der Magenverdauung) kann in diesen Fällen gar nicht die Rede sein; ebensowenig von dem Auskratzen (Bernays) oder Einschneiden der Narbe (Ceccerelli) von einer Magenwunde aus.

Bei der Nachbehandlung ist auf die bekannten diätetischen Vorschriften und ganz besonders auf die Belandlung der Magenerweiterung Gewicht zu legen. Sind die Magenwände durch die Dilatation schlaff und und unfähig, sich zu contrahiren, dann ist auch die Gefahr einer Wiederbildung der Verengerung gegeben, diese erschwert das Zurückgehen der Ektasie, und so bildet sich ein sehr schlimmer Circulus vitiosus. Oft genug war die Leistungsunfähigkeit der Magenmuskulatur Ursache des bösen Ausganges nach verschiedenen Operationen am Magen; die Verengerung am Pylorus \%. B. war durch Resection, Dilatation oder Plastik beseitigt, oder es war ein neuer Magenausgang durch Jejunogastrostomie geschaffen; der neue Canal hatte eine ausreichende Weite - aber der Mageninhalt ging nicht hindurch, die Inanition blieb, der Kıanke starb trotz der Operation. Vielleicht lässt sich dieses Ereigniss vermeiden, wenn man nach den ersten 3, 4 Tagen, in denen wenig oder nichts geschluckt und ernährende Clysmata gegeben werden, 4 bis 5 Stunden nach jeder Mahlzeit den Magen vorsichtig aushebert, sobald sich die Zeichen mangelhafter Rüickbildung der Ektasie einstellen. Mau würde dadurch einer Anhäufung von Speisebrei, einer Zersetzung und Gasbildung, und dadurch einer Verschlimmerung des Zustandes vorbeugen. Selbstverständlich müssen die Kranken vernünftig sein und ihren schwachen Magenwänden nicht Leistungen zumuthen, wie z. B. die Verarbeitung von Wurststïcken, Apfelsinen und anderen halb unverdaulichen Dingen.

Bei denjenigen Fällen von Pyloroplastik, von denen wir sagten, sie seien trotz der Operation gestorben - Fälle, wie sie nach anderen Operationen am Bauche noch viel häufiger beobachtet werden muss sich jeder fragen, ob nicht bei früherem Operiren ein günstiges Resultat erzielt wäre. Diese Frage ist unendlich wichtig für die Praxis, aber leider vorläufig nicht zu beantworten. Unbestimmte und allgemeine Vorschriften, wie die, dass man operiren müsse, so lange der Kranke noch leidlich kräftig sei u. s. w., haben wenig Werth, und bestimmte Anhaltspunkte bietet weder der allgemeine Eindruck, del der Kranke macht, noch im speciellen die Beschaffenheit des Pulses, die Häufigkeit des Erbrechens, die Urinnienge u. s. w. Jeder Arzt wird Fälle beobachtet haben, in denen höchst bedrohliche Symptome vorhanden waren, in denen alles verloren schien, und in denen trotz alledem eine spontane Rückbildung der Ektasie, dieses Hauptsymptoms der Pylorusenge, und eine vollständige Wiederherstellung des Kranken eiutrat. Diese Erfahrung, sowie die Thatsache, dass man z. B. bei Anätzungen auch nicht annähernd die Zeit bestimmen kann, in welcher der Substanzverlust soweit verheilt ist, dass man nicht mehr an kranken ulcerösen und erweichten Stellen schneiden und leider dann auch nähen muss, sprechen gegen eine frühe Operation in diesen Fällen. Bei Stenosen infolge der Vernarbung anderer Geschwüre wird man, wenn sich bei der Spaltung des Pylorus die Unmöglichkeit der Plastik herausstellt, nach Schluss der Wunde die Jejunogastrostomie anschliessen können; es ist zweifellos, dass dies bei ausgedehnten Anätzungen nicht immer möglich ist. Wird diese Aetiologie nicht zugegeben - sie kann, wie wir oben erwähnt haben, trotzdem vor- handen sein - handelt es sich mit Wahrscheiulichkeit urn die einfache sogenannte Hypertrophie des Pylorus, dann wird man bei der Gefahrlosigkeit der Laparotomie und Pyloroplastik an und für sich nicht auf gefahrdrohende Symptome warten, sondern operiren, wenn regelmässige Magenausspülungen, und dadurch Entleerung des erweiterten Magens nicht bald einen deutlichen Erfolg durch Abnahme der Ekktasie, Besserung der Abmagerung und Schwäche, $\mathrm{Zu}$ nahme des 24 stïndigeu Urinquantums haben.

Zum Schluss spreche ich den Herren, welche anf meine Anfragen in liebenswürdigster Weise mir über die voll ihnen operirten Fälle Auskunft ertheilt und mir dadurch die vorstehenden Betrachtungen überhaupt ermöglicht haben, Herren Geh.-Rath Mikulicz, Prof. Heineke, Dr. van der Hoeven, Lauenstein und Ortmann, ganz besonders Herrn Prof. Novaro (Siena) meinen besteu Dank aus.

Litteratur (ansser der in der Tabelle angegebenen).

Codivilla, Gazz. d. osp. 75 u. 78/88 (Eine IIeilung durch Lösung von Adhäsionen, eine nach zweimaliem Dehnen des hypertrophischen Pylorns von einer Magenwunde aus). - Poggi, Ital. Chir. Congr., s. Deutsche med. Wochenschr. 1889, No. 2 (2 Fälle von Divulsion des narbigen Pylorus nach Loreta). - Treves, Brit. med. Journ. 18. Mai 1889 (Eine Heilung durch die Operation nach Loreta, noch nach einem Jalre beobachtet). - Kinnicut und Bull, New-York Rec. Jini 1889, p. 617 (ebenfalls Loreta, Heilıng nach einem Jahre beobachtet). - Barton, ebenda p. 585, Bd. 35, No. 21 (Eine Heilung nach Loreta). (Dasselbe: Med. News, 25. Mai 1889 Digital divulsion of the pylorus for cicatr. stenosis.) - Huntington, Occid. med. Times Sept. 1889, ref. im Centralbl. f. Chir. 1890, No. 5 (Eine Heilung nach Loreta). - Gardner, Brit. med. Journ. 14. Dec. 1889 (Eine Heilung nach Loreta). - Angerer, Arcl. f. klin. Chir. Bd. 39, Heft 2 (Ueber die Diagnose und Indication zum Operation der Pylorusstenose). - Kl emperer, Deutsche med. Wochenschr. 1889, No. 9 (1 Fall von geheilter Magendilatation, Fall 7 der Tabelle). - Schmitz, Dissert. Berlin 1889 (Zur Casuistik der Operationen der Pylorisstenose). - Bernays, Ann. of Surg. Dec. 1887 (Auskratzen nach Gastrotomie) 